\title{
Weiterbildung schützt Patienten
}

Die Publikation Editors' Choice: Impact of provider characteristics on use of endovenous ablation procedures in Medicare beneficiaries im Journal of Vascular Surgery Venous and Lymphatics, März 2019, von den Autoren: John T. Baber Jr, Jialin Mao, Art Sedrakyan, Peter H. Connolly, and Andrew J. Meltzer, aus New York und Scottsdale bestätigt sicherlich bei einigen von uns, was wir befürchtet haben... (J Vasc Surg: Venous and Lym Dis. 2019;7:203-9.) Die Arbeit ist erfrischend ehrlich und kritisch und das macht sie dadurch besonders beachtenswert. Denn gerade das, worüber man sich hierzulande manchmal ärgert, Weiterbildungserfodernisse, Kontrollen, Restriktionen, schützt vor dem, was beschrieben wird.

Das Ziel der Studie war es, den Zusammenhang zwischen den Charakteristika der Anbieter endovenös thermischer Verfahren (EVT) und der Häufigkeit der Anwendung bei den Versicherten einer Krankenkasse zu analysieren.

Die Daten der Medicare Versicherung (USA) zwischen 2012-2014 wurden in eine Datenbank eingespeist mit folgenden Kriterien: Anwender von Laser oder Radiofrequenz zur Behandlung der Varikose wurden gelistet und aufgeteilt nach ihrer Fachrichtung, Art der Behandlung (stationär oder ambulant), der geographischen Region für jeden Patienten. Es wurde ein Nutzungsindex erstellt, der die Anzahl der EVT Prozeduren je Patient und Jahr erfasste. Er bezieht sich auf die Anzahl der behandelten Venen je Patient und Jahr - unabhängig davon, ob sie in einer Sitzung oder mehreren angegangen wurden. Bei einer behandelten Vene liegt er bei 1, bei Behandlung von beiden V. saphena magnae in einem Jahr bei 2, der Höchstwert lag bei 4 ( $2 \times$ VSM, $2 \times$ VSP). Mittels multivariater Regressions-Analyse wurden die Charakteristika der Anwender mit einem Nutzungs-Index über dem 75.-ten Perzentil herausgearbeitet.
Als Ergebnis wurden 6.599 Anwender identifiziert, die EVT Prozeduren an 405.232 Medicare Versicherten angewendet hatten. Die Hälfte der Prozeduren wurde im Süden der USA durchgeführt. 23 \% der Anbieter waren Gefäßchirurgen, sie behandelten $20 \%$ der Fälle. $19 \%$ waren Kardiologen und behandelten $20 \%$ der Fälle. Allgemein-Chirurgen machten 13,5\% der Anbieter aus und versorgten $17 \%$ der Fälle. 9,3\% der Anbieter waren Radiologen ( $8 \%$ der Fälle), 7,7\% gehörten zu anderen chirurgischen Spezialisierungen ( $8 \%$ der Fälle) und $30 \%$ waren „andere Spezialisierungen “ mit $27 \%$ der Fälle, wobei hierzu auch Pathologen und Augenärzte zählten. Somit war die Anzahl der Fälle je Fachgruppe relativ homogen.

Gefäßchirurgen hatten jedoch den niedrigsten Nutzungsindex mit 1,32 Prozeduren je Patient und Jahr. Somit behandeln spezialisierte Ärzte weniger Venen je Patient als nicht spezialisierte. Mittels multivariater Analyse konnte errechnet werden, dass die Wahrscheinlichkeit, dass ein Anwender einen Nutzungsindex über 1,8 hatte (oberste $25 \%$ ) damit zusammen hing, dass er kein Facharzt für Chirurgie, Kardiologie oder Radiologie war (Odds ratio (OR): 3,35, 2,74$4,09)$, dass es sich um eine ambulante Einrichtung handelte (OR: 2,62, 1,97-3,47), bzw. um einen Anbieter, der insgesamt eine hohe Anzahl an Eingriffen durchführte (dessen Eingriffsanzahl im oberen 75 Perzentil lag) (OR: 8,68; 7,59-9,91).

Die Autoren schlussfolgern, dass es eine große Variation in Bezug auf die Intensität der venösen Ablation in Abhängigkeit des Wohnortes und der Spezialisierung des Anbieters gibt. Außerdem scheinen Anbieter mit einer hohen Anzahl an Behandlungen pro Jahr und solche, deren Fachbereich nicht üblicherweise chronische venöse Insuffizienz der unteren Extremität beinhaltet, mehr Eingriffe je Patient und Jahr durchzuführen.
Einschränkungen: Leider ist nicht zu ersehen, ob die mehrfachen Eingriffe an einem Tag oder nacheinander, an derselben Vene oder an unterschiedlichen durchgeführt wurden. Der Schweregrad der Erkrankung ist nicht ablesbar, es handelt sich um Versicherte einer Versicherung - und nicht um eine Querschnittstudie. Dennoch ist klar zu sehen, dass die Anzahl der behandelten Venen je Patient bei einem höheren phlebologischen Kenntnisgrad - wie es den Gefäßchirurgen zuzuordnen wäre - am geringsten ist.

Anwendbarkeit auf unsere Situation in Deutschland: Je besser weitergebildet der behandelnde Arzt, desto weniger Eingriffe je Patient und Jahr wurden durchgeführt. Das kann daran liegen, dass die phlebologischen Kenntnisse ein selektiveres Identifizieren der zu behandelnden Vene besser ermöglicht und damit die Planung der Strategie besser ist, dass Gefäßmediziner um den Wert des Venenerhalts als Bypass-Material wissen und/oder dass die nicht phlebologisch vorgebildeten Ärzte, die diese Verfahren anwenden, dies eher aus pekuniären Aspekten tun, weil das Verfahren einfach zu erlernen und im Vergleich zu anderen Verfahren anderer Fachrichtungen lukrativ ist.

Dies wäre - dank der Reglementierungen! - in Deutschland nicht möglich. Es ist erfreulich, dass die Krankenkassen bei den meisten Integrierten Versorgungsverträgen, über die die endoluminal thermischen Verfahren hierzulande bei den meisten Patienten bezahlt werden, sich an das PhlebologieFortbildungszertifikat halten als Garant für eine einschlägige Weiterbildung. Und es ist sehr gut, dass die Zusatzbezeichnung Phlebologie erhalten blieb und wir weiterhin für Weiterbildung und Fortbildung sorgen können und somit hoffentlich zur guten Qualität in der Versorgung beitragen können!

Erika Mendoza 